\title{
Individual Responsibility and Recovering Nature in a Post- Pandemic World
}

\author{
Shahino Mah Abdullah*
}

The COVID-19 pandemic has severely impacted public health and the global economy. It has likewise affected the environment: plastic pollution has increased significantly due to littered disposable protective gear, especially in streets, drains, rivers, and oceans. Littered protective gear has been observed piling up in rubbish dumps, clogging the flow of water, inviting pests, creating uncomfortable scenery, spreading various diseases, and harming marine life. This situation will be detrimental to the people living in such environments. For this reason, green economic recovery strategies are necessary to conserve the environment while keeping up with the post-pandemic economic recovery. To begin with, the root cause of the problem must be clearly identified so that reset, restore, and recovery measures can be well implemented.

First, it is crucial to note that preserving the environment is the responsibility of all sections of society, including individuals, NGOs, industry players, and the government. Currently, the main offenders causing the random littering of used masks and gloves are those irresponsible individuals who take environmental preservation for granted. Plastic pollution caused by disposable protective gear has created various problems, ranging from environmental to economic to health, and will eventually cost many lives. At this individual level, a reset measure can be initiated by inculcating an awareness that such used items and other waste must be disposed of properly. Any effort to change individual behaviour is always continuous, however, as evinced by a series of decades-long global awareness campaigns aimed at educating individuals about proper waste disposal.

The 3Rs (Reduce, Reuse, Recycle) campaign, for example, has been in place for a long period of time, with almost everybody now knowing about it. It has been introduced in schools and widely publicised during Earth Day. The effectiveness of such campaigns, however, has been varied, both from region to region and country to country, with few having achieved effective implementation. Indeed, most have not achieved their expected minimum targets, with a lack of consistency being a key factor in their failure. This situation provides a clear idea of the importance associated with addressing pre-pandemic issues so as to ensure reset, restore, and recovery measures are on the right track to begin with. From now on, therefore, we have to seek a post-pandemic solution capable of solving previous issues, as well as those that have emerged after the pandemic. 
It is important that any solution be sustainable so that it can solve both past and current environmental issues, while continuing into the future to help preserve nature in the long run.

As resetting measures must start at the individual level, it is important to recognise the impact of individual behaviour on handling waste. How individuals take care of waste varies, but can be divided into three groups. The first group can be categorised as reckless polluters, or the main actors responsible for littering. They comprise those who arbitrarily litter waste (used masks and gloves), no matter where they are. Those individuals who sometimes litter due to a lack of disposal facilities also fall under this category since they do not fully dispose of their waste properly. The second group can be categorised as responsible individuals who dispose of used masks and gloves in the right way, whether into a recycle bin or other dedicated receptacle, including a general waste bin. The last group is then an extension of this second group, consisting of those who willingly do more than just manage their own waste, such as voluntarily collecting and disposing that of others, especially from the first group. The individuals in this last group have embraced 'sustainable behaviour', an integral element for recovering both the economy and the environment post-pandemic. In brief, sustainable behaviour encompasses ecological, selfless, prudent, and equitable actions that result in the conservation of natural and social resources. It has a significant impact on saving the environment while also recovering the economy of a country.

By grouping individuals based on their waste management, proper and suitable awareness can be produced to enrich behaviour. For instance, individuals in the first group must be encouraged to change their habits and act more responsibly so that they will dispose of used masks and gloves at least in a waste bin. A creative message and initiative to change their attitude is necessary because, ultimately, the world needs everyone to be in at least the second group, to be responsible for what they have and manage. Preferably, however, they should also develop the same awareness as those in the last group, who voluntarily manage the waste of others. Such people always have a chance to make a better world because they have the character to keep away from polluting the environment. The number of those in the third group will increase through the right awareness raising strategies. For those who achieve such a level of awareness, they should be supported and valued so that they will continue their voluntary efforts. Inculcating awareness might seem a simple method to improve individual behaviour, but it will allow a stimulation of sustainable behaviour that will play a major role in restoring the environment post-pandemic.

Awareness campaigns, however, do not always change the behaviour of individuals. Strict measures by the authorities, including fines and other 
punishments, are also sometimes necessary. However, the enforcement of such measures can also be ineffective and troublesome. Irresponsible individuals might see a fine, for example, as a cheap consequence of their wrongdoing and, sadly, still have no awareness. There are also cases where such individuals escape being traced and punished after violating the rules. Therefore, alternative measures are also needed. This requires us to go back to the root cause of the problem: that the pollution originates from littered masks, gloves, and other protective gear. These are all disposable items designed for single use only; the more people use them, the more chance there is of pollution due to irresponsible individuals. Therefore, a shift from single use to reusable is necessary to significantly reduce plastic pollution. A single washable mask that can be used for a month, for example, will eliminate reliance on disposable masks and reduce the chances of littering, thus saving the environment.

\section{Notes}

* Shahino Mah Abdullah is a Research Fellow at the International Institute of Advanced Islamic Studies (IAIS) Malaysia. He can be contacted at: shahino@ iais.org.my. 